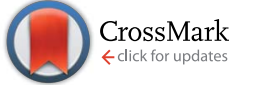

Cite this: Chem. Sci., 2016, 7, 2371

Received 11th August 2015

Accepted 17th December 2015

DOI: $10.1039 / \mathrm{c} 5 \mathrm{sc} 02978 \mathrm{c}$

www.rsc.org/chemicalscience

\section{Quillaja saponin variants with central glycosidic linkage modifications exhibit distinct conformations and adjuvant activities $\uparrow$}

\author{
William E. Walkowicz, ${ }^{a}$ Alberto Fernández-Tejada, ${ }^{\text {bc }}$ Constantine George, ${ }^{d}$

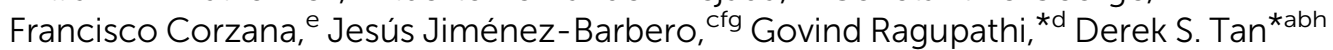 \\ and David Y. Gintabh
}

Immunological adjuvants such as the saponin natural product QS-21 help stimulate the immune response to co-administered antigens and have become increasingly important in the development of prophylactic and therapeutic vaccines. However, clinical use of QS-21 is encumbered by chemical instability, doselimiting toxicity, and low-yielding purification from the natural source. Previous studies of structureactivity relationships in the four structural domains of QS-21 have led to simplified, chemically stable variants that retain potent adjuvant activity and low toxicity in mouse vaccination models. However, modification of the central glycosyl ester linkage has not yet been explored. Herein, we describe the design, synthesis, immunologic evaluation, and molecular dynamics analysis of a series of novel QS-21 variants with different linker lengths, stereochemistry, and flexibility to investigate the role of this linkage in saponin adjuvant activity and conformation. Despite relatively conservative structural modifications, these variants exhibit striking differences in in vivo adjuvant activity that correlate with specific conformational preferences. These results highlight the junction of the triterpene and linear oligosaccharide domains as playing a critical role in the immunoadjuvant activity of the Quillaja saponins and also suggest a mechanism of action involving interaction with a discrete macromolecular target, in contrast to the non-specific mechanisms of emulsion-based adjuvants.

\section{Introduction}

Classical vaccines based on whole live or attenuated pathogens have been used to prevent a variety of life-threatening infectious diseases. In contrast, many modern subunit vaccines now being developed for both prophylactic and therapeutic applications in a variety of diseases use defined molecular antigens to generate

${ }^{a}$ Louis V. Gerstner, Jr. Graduate School of Biomedical Sciences, 1275 York Avenue, New York, NY 10065, USA

${ }^{b}$ Chemical Biology Program, Memorial Sloan Kettering Cancer Center, 1275 York Avenue, New York, NY 10065, USA

${ }^{c}$ Chemical \& Physical Biology, Center for Biological Research, CIB-CSIC, 28040 Madrid, Spain

${ }^{d}$ Melanoma \& Immunotherapeutics Service, Department of Medicine, Memorial Sloan Kettering Cancer Center, 1275 York Avenue, New York, NY 10065, USA

${ }^{e}$ Department of Chemistry, University of La Rioja, 26006 Logroño, Spain

${ }^{f}$ Structural Biology, Center for Cooperative Research, CIC-bioGUNE, 48160 DerioBizkaia, Spain

${ }^{g}$ Ikerbasque, Basque Foundation for Science, 48013 Bilbao, Spain

${ }^{h}$ Tri-Institutional Research Program, Memorial Sloan Kettering Cancer Center, 1275 York Avenue, New York, NY 10065, USA. E-mail: tand@mskcc.org

$\dagger$ Electronic supplementary information (ESI) available. See DOI: $10.1039 / \mathrm{c} 5 \mathrm{sc} 02978 \mathrm{c}$

† Deceased March 22, 2011. more targeted and effective responses. However, many of these molecular antigens are poorly immunogenic, requiring additional stimulation of the immune system for vaccine efficacy. ${ }^{\mathbf{1}}$

Accordingly, immunological adjuvants that help stimulate the immune response to antigens with which they are coadministered $^{2}$ have become critically important in the development of vaccines against infectious diseases, neurodegenerative disorders, and cancer. ${ }^{3}$ Immunoadjuvants can increase the effectiveness of vaccines that use poorly immunogenic antigens and enable dose-sparing of expensive or rare antigens. The current clinically-approved adjuvants alum, ${ }^{4} \mathrm{AS} 04,{ }^{5} \mathrm{MF} 59,{ }^{6}$ and $\mathrm{AS} 3^{7}$ are emulsions that stimulate an immune response through several pathways of the innate immune system, including non-specific sensors of inflammation ${ }^{6 a}$ (NLRP3 inflammasome) and pathogen-associated molecular patterns ${ }^{8}$ (e.g., monophosphoryl lipid A). Such adjuvants effectively stimulate antibody-mediated Th2-type immunity and have been used extensively since the discovery of the immunopotentiating properties of aluminum salts in 1926, in vaccines against toxins and viruses such as diphtheria-tetanus-pertussis, hepatitis B, and hepatitis A. $^{4 b}$ More recently, emulsion-based adjuvants have been used in prophylactic vaccines against human papilloma virus ${ }^{9}$ as well as seasonal ${ }^{7}$ and pandemic $^{10}$ influenza viruses. However, efforts to develop therapeutic and 
prophylactic vaccines for other diseases (cancer, HIV infection, malaria, herpes, tuberculosis) have achieved limited success, due in large part to poor antigen immunogenicity and insufficient cell-mediated Th1-type immune responses. ${ }^{1}$

The saponin natural product immunoadjuvant QS-21 potently stimulates both Th1 and Th2 responses and has been the experimental immunoadjuvant of choice for two decades. ${ }^{11}$ Isolated from the bark of the Quillaja saponaria Molina tree, QS21 has shown promising results in dozens of vaccine clinical trials, ${ }^{12}$ including a recently reported breakthrough study on the prevention of childhood malaria in Africa. ${ }^{13}$ Unfortunately, QS21 suffers from several liabilities including chemical instability at room temperature, which hinders vaccine deployment in the developing world $^{\mathbf{1 4}}$ dose-limiting toxicity involving local erythema and inflammation as well as systematic flu-like symptoms, ${ }^{12,15}$ and low-yielding (0.001\%) purification from the natural source, ${ }^{11 a}$ potentially complicated by metabolomic heterogeneity of $Q$. saponaria bark extracts. ${ }^{\mathbf{1 6}}$ In addition, its mechanism of action remains poorly understood. ${ }^{\mathbf{1 7}}$ To address these limitations, we have previously completed the first total synthesis $^{18}$ of QS-21 and also developed a modular semisynthetic strategy from partially purified $Q$. saponaria extracts ${ }^{\mathbf{1 9}}$ that has provided access to dozens of analogues, yielding key insights into saponin structure-activity relationships (SAR).

The structure of QS-21 (1) can be divided into four domains: a branched trisaccharide, a quillaic acid triterpene, a bridging linear oligosaccharide, and a pseudo-dimeric acyl chain (Fig. 1a). The primary constituents of natural QS-21 extracts are $\mathrm{a} \approx 2: 1$ mixture of xylose (1a) and apiose (1) isomers at the terminus of the linear oligosaccharide domain. We have previously found that simplified acyl chain variants retain in vivo adjuvant activity and exhibit reduced toxicity relative to the natural product. ${ }^{20}$ Subsequently, stepwise truncation of the right-hand bridging linear tetrasaccharide identified trisaccharide variant 2 (SQS-0-0-5-5) (Fig. 1b) as having an optimum balance of activity and synthetic accessibility, albeit with reemergence of toxicity. ${ }^{21}$ In recent work, carried out concurrently with this study, we discovered that the entire left-hand branched trisaccharide domain is dispensable for adjuvant activity and also investigated the importance of the C4-aldehyde and C16-hydroxyl substituents in the triterpene domain. ${ }^{22}$

Herein, we report our investigations of the most prominent unexplored structural feature of the Quillaja saponaria (QS) saponins, the central glycosyl ester linkage that connects the triterpene and the linear oligosaccharide domains. Based on the previously identified lead structure 2 (SQS-0-0-5-5), ${ }^{21}$ we synthesized a series of linkage variants to modulate the distance and orientation of the two domains. Construction of these variants in the context of the complex saponin structure proved challenging, requiring formation of sterically-encumbered glycosidic bonds without the aid of neighboring group participation. As a result, several of the linkages were formed using unconventional glycosylation promoters. In a preclinical mouse vaccination model, these linkage variants exhibited a remarkable range of adjuvant activities and toxicities, providing new insights into the structural requirements for adjuvant activity in the QS saponin class. Molecular dynamics simulations of these synthetic variants and the major natural product isomer QS-21Api (1a) revealed distinctive conformational preferences that correlate with observed adjuvant activities.

\section{Results and discussion}

\section{Design of QS saponin variants with modified central glycosidic linkages}

At the outset, we envisioned that a series of linkage variants could be used to probe the effects of distance, stereochemistry, and conformational flexibility upon adjuvant activity (Fig. 1b). The $\beta$-glycosyl ethanolamide $\mathbf{3}, \boldsymbol{\beta}$-glycosyl carbamate $\boldsymbol{\beta}$-4, and $\beta$ glycosyl thioester $\mathbf{5}$ were designed to increase the distance between the triterpene and trisaccharide domains. Meanwhile, the $\alpha$-glycosyl ester $6, \alpha$-glycosyl amide 7 , and $\alpha$-glycosyl carbamate $\boldsymbol{\alpha} \mathbf{- 4}$ were designed to probe the importance of stereochemistry at this glycosidic linkage. Finally, the $\beta$-glycosyl ether 8 and $\beta$-glycosyl thioether $\mathbf{9}$ were designed to probe the effect of increasing conformational flexibility between the two domains. Notably, these linkages would need to be assembled in the context of the complex saponin framework, which features sterically congested aldehyde and carboxylic acid functionalities, as well as numerous, previously established protecting groups that are incompatible with strongly acidic or basic conditions, limiting the range of potential functional group manipulations and glycosylation conditions that could be used.

\section{Synthesis of glycosyl acceptors from the C28 carboxylic acid of a protected prosapogenin}

The syntheses of these variants began with functionalization of the protected prosapogenin 10, abbreviated herein as PPS, to provide the corresponding glycosyl acceptors (Scheme 1). ${ }^{19}$ The C28-carboxylic acid was treated with diphenylphosphoryl azide to give an acyl azide intermediate, which, upon continued heating, underwent Curtius rearrangement to isocyanate $\mathbf{1 1}$. Alternatively, activation of the C28-carboxylic acid in $\mathbf{1 0}$ with thionyl chloride proceeded in quantitative yield to afford the bench-stable acyl chloride 12, which was readily functionalized with a variety of nucleophiles, including ethanolamine to form ethanolamide 13, and ammonia to provide primary amide $\mathbf{1 4}$. While addition of nucleophiles such as $\mathrm{NaSH}$ and 9-fluorenylmethylthiol furnished the corresponding thioacid and thioester products in poor yields, these proved recalcitrant to purification and were not advanced further. Access to neopentyl alcohol 15 required careful chemoselective reduction of acyl chloride 12 with tetrabutylammonium borohydride. Extended reaction times and other hydride reductants (DIBAL-H, Red-Al, $\mathrm{NaBH}_{4}$, super-hydride) led to overreduced and desilylated byproducts.

Conversion of this sterically encumbered alcohol $\mathbf{1 5}$ to the corresponding thiol 16 also proved challenging. Tosylation or mesylation was sluggish and the resulting sulfonate derivatives were completely unreactive to thiol nucleophiles. Ultimately, we resorted to conversion of alcohol 15 to the corresponding triflate, which proved to be a competent electrophile for substitution with potassium thioacetate in the presence of 18-crown-6 
a) QS-21 natural product

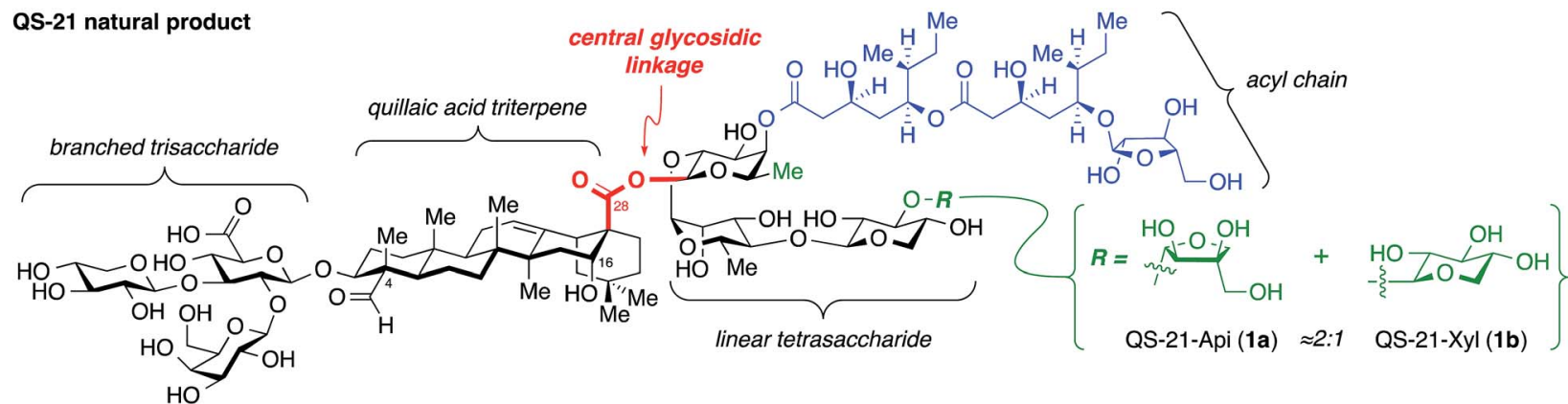

b) Semisynthetic QS saponin analogues

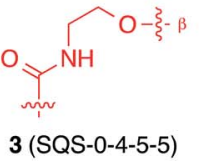

3 (SQS-0-4-5-5) ethanolamide
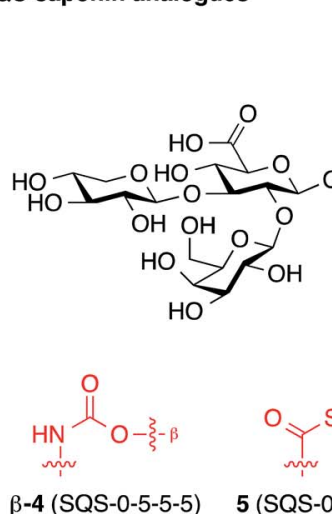

B-glycosyl
(SQS-0-5-5) carbamate

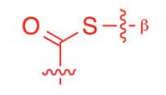

5 (SQS-0-13-5-5) $\beta$-glycosyl thioester

linker length variants

(1)<smiles>C1CC2CCC1O2</smiles>

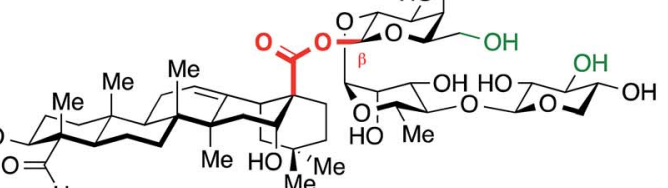
$\mathrm{HO} \mathrm{Me}$

2 (SQS-0-0-5-5) $\beta$-ester

Fig. 1 (a) Structure of the saponin natural product immunoadjuvant QS-21 ( $\approx 2: 1$ mixture of 1a/1b). (b) Structures of a simplified semisynthetic lead compound 2 and corresponding glycosidic linkage variants 3-9 designed to probe the effects of linker length, stereochemistry, and conformational flexibility upon adjuvant activity. Four-number SQS (synthetic QS) codes designate structural variants in each of the four corresponding structural domains of QS-21, left to right, with 0 assigned to the natural product structure.

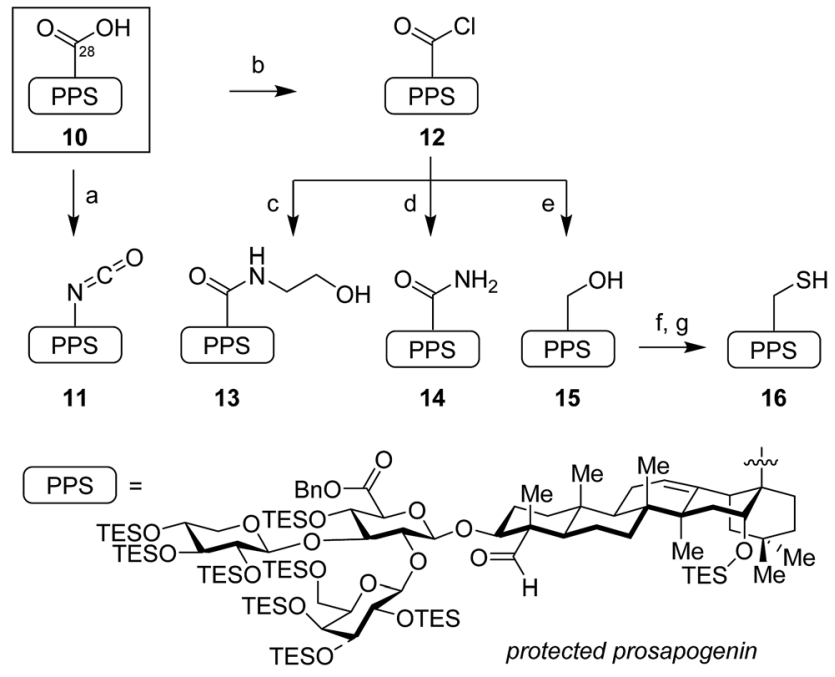

Scheme 1 Synthesis of glycosyl acceptors from protected prosapogenin (PPS). Reagents and conditions: (a) $(\mathrm{PhO})_{2} \mathrm{P}(\mathrm{O}) \mathrm{N}_{3}, \mathrm{Et}_{3} \mathrm{~N}, \mathrm{PhH}$, $80{ }^{\circ} \mathrm{C}, 79 \%$; (b) $\mathrm{SOCl}_{2}$, pyr, $\mathrm{CH}_{2} \mathrm{Cl}_{2}, 0{ }^{\circ} \mathrm{C}, 99 \%$; (c) $\mathrm{HO}\left(\mathrm{CH}_{2}\right)_{2} \mathrm{NH}_{2}$, $\mathrm{CH}_{2} \mathrm{Cl}_{2}, \mathrm{O}^{\circ} \mathrm{C}, 88 \%$; (d) $\mathrm{NH}_{3}, \mathrm{CH}_{2} \mathrm{Cl}_{2}, 0^{\circ} \mathrm{C}, 92 \%$; (e) $\mathrm{Bu}_{4} \mathrm{NBH}_{4}, \mathrm{CH}_{2} \mathrm{Cl}_{2}$, $0{ }^{\circ} \mathrm{C}, 87 \%$; (f) $\mathrm{Tf}_{2} \mathrm{O}$, pyr, $\mathrm{CH}_{2} \mathrm{Cl}_{2}, 0{ }^{\circ} \mathrm{C}$; AcSK, 18-crown-6, $1: 1 \mathrm{THF} /$ DMF, $0{ }^{\circ} \mathrm{C}$, 92\%; (g) $\mathrm{H}_{2} \mathrm{NNH}_{2}$, DTT, $1: 1 \mathrm{THF} / \mathrm{DMF}, 90 \%$. DTT = dithiothreitol; PPS = protected prosapogenin. ether. Treatment of the resulting thioacetate with hydrazine under reducing conditions (DTT) furnished the desired prosapogenin thiol 16 in excellent yield (83\% over two steps).

\section{Construction of glycosidic linkages using the linear trisaccharide glycosyl donor as an electrophile}

While glycosylation reactions to form all of the linkage chemotypes targeted herein are well-documented in the literature, synthesis of glycosyl linkages with this level of complexity and in sterically demanding environments are limited in many cases (amide, ${ }^{23}$ thioether ${ }^{24}$ ), and apparently without precedent in another (thioester). Our initial efforts began with the previously described trisaccharide hemiacetal $17,{ }^{21}$ which successfully underwent dehydrative glycosylation $\left(\mathrm{Ph}_{2} \mathrm{SO} / \mathrm{Tf}_{2} \mathrm{O}\right)$ with prosapogenin ethanolamide $\mathbf{1 3}$ to give the ethanolamide-linked intermediate 18 in excellent yield (87\%) and with complete $\beta$ selectivity (Scheme 2).

In contrast, glycosylation of prosapogenin primary amide $\mathbf{1 4}$ required extensive optimization of reaction conditions to achieve anomeric selectivity. Ultimately, we found that use of a twofold excess of the glycoside acceptor $\mathbf{1 4}$ favored formation of $\boldsymbol{\beta}$ 19 (2:1 to $4: 1 \beta / \alpha, 60-78 \%$ combined yield of separable anomers). Notably, the anomeric preference could be reversed 


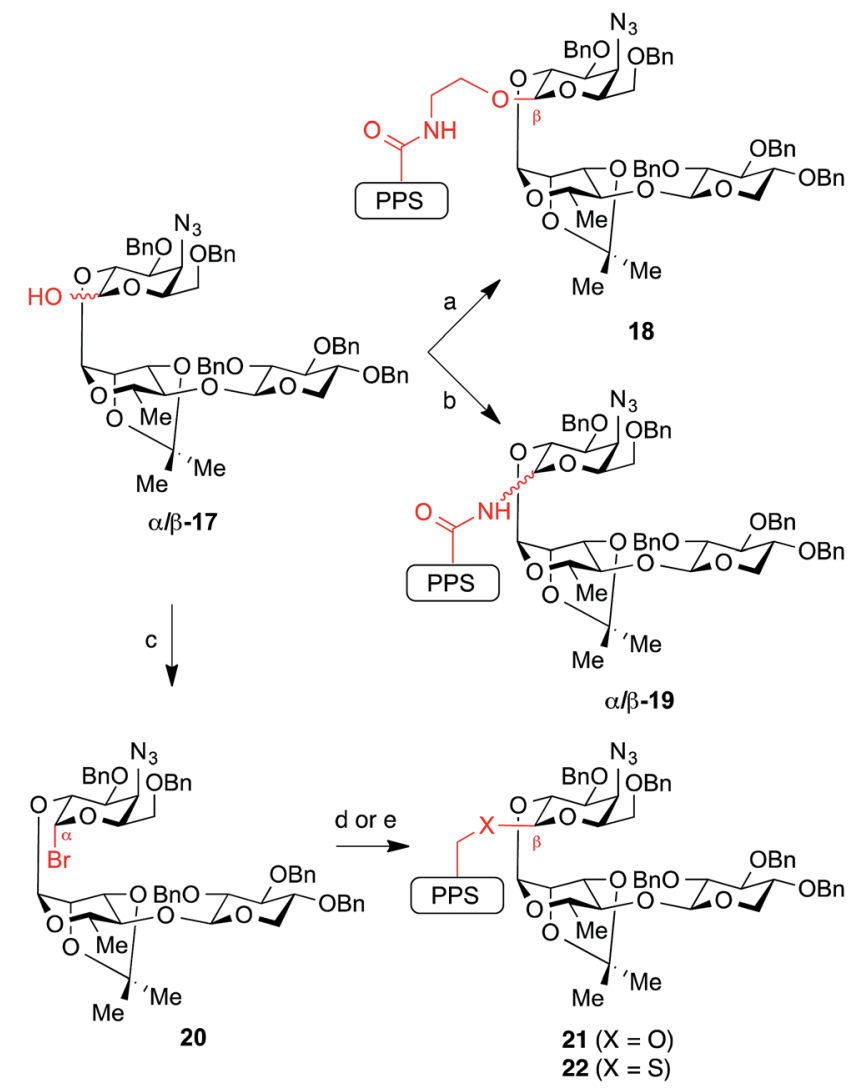

Scheme 2 Synthesis of glycosidic linkage variants via traditional glycosylations using the glycosyl donor as an electrophile. Reagents and conditions: (a) $\mathrm{Ph}_{2} \mathrm{SO}, \mathrm{Tf}_{2} \mathrm{O}, \mathrm{TBP}, \mathrm{CH}_{2} \mathrm{Cl}_{2},-45^{\circ} \mathrm{C}$, then add 13 , 87\% ( $\beta$ only); (b) $\mathrm{Ph}_{2} \mathrm{SO}, \mathrm{Tf}_{2} \mathrm{O}, \mathrm{TBP}, \mathrm{CH}_{2} \mathrm{Cl}_{2},-45^{\circ} \mathrm{C}$, then add $14,60-$ $78 \%$ combined yield of separable anomers (2 equiv. $14 \rightarrow 2: 1$ to $4: 1$ $\boldsymbol{\beta} / \boldsymbol{\alpha}-19 ; 2$ equiv. $17 \rightarrow 1: 6 \boldsymbol{\beta} / \boldsymbol{\alpha}-19) ;(\mathrm{c})(\mathrm{COBr})_{2}, \mathrm{DMF}, \mathrm{TBP}, \mathrm{CH}_{2} \mathrm{Cl}_{2}$, $0{ }^{\circ} \mathrm{C}, 74 \%$; (d) $\mathrm{X}=\mathrm{O}$ : 15 , AgOTf, TBP, $\mathrm{CH}_{2} \mathrm{Cl}_{2},-40{ }^{\circ} \mathrm{C}, 69 \%$ isolated yield of $\beta$-anomer ( $>20: 1 \beta / \alpha$ ratio in crude product); (e) $X=S: 16$, $\mathrm{NaH}, 2.5: 1 \mathrm{THF} / \mathrm{DMF}, 0{ }^{\circ} \mathrm{C}, 69 \%$. TBP $=2,4,6$-tri-tert-butylpyridine.

to favor $\alpha-\mathbf{1 9}$ when a two-fold excess of the donor $\mathbf{1 7}$ was used ( $6: 1 \alpha / \beta, 71 \%$ combined yield). Unfortunately, while these anomers were separable, the $\beta$-glycosyl amide linkage in $\boldsymbol{\beta}-\mathbf{1 9}$ proved surprisingly acid-sensitive, and was cleaved under even mildly acidic conditions (e.g., $\left.\mathrm{SiO}_{2}, \mathrm{HF} \cdot \mathrm{pyr}, \mathrm{AcOH}\right)$. Despite considerable efforts, global deprotection could not be achieved due to competing cleavage of the glycosidic $\mathrm{C}-\mathrm{N}$ bond under the reaction conditions $\left(3: 1 \mathrm{TFA} / \mathrm{H}_{2} \mathrm{O}\right)$ to reform the primary amide 14 and hemiacetal 17. In contrast, a corresponding $\beta$ glycosyl pivalamide model system ${ }^{25}$ was significantly more stable $\left(\mathrm{SiO}_{2}\right)$, highlighting the complexities of working with the complete saponin scaffold. Unlike its $\beta$-anomer, the axiallydisposed glycosyl amide in $\alpha-19$ showed no observable acid lability, illustrating the significant changes in reactivity that may arise from minor structural perturbations of the central glycosidic linkage.

We next sought to prepare the glycosyl ether linkage in 21. To our surprise, repeated attempts at glycosylation of prosapogenin neopentyl alcohol $\mathbf{1 5}$ with the same trisaccharide hemiacetal donor $\mathbf{1 7}$ under dehydrative or Schmidt conditions gave no isolable glycosylation products. However, we found that the glycosylation could be effected smoothly with trisaccharide bromide donor 20 (prepared from hemiacetal 17 with oxalyl bromide, DMF) under the silver triflate-promoted KoenigsKnorr reaction at low temperature to give the ether-linked intermediate 21 with $>20: 1 \beta / \alpha$ selectivity.

The analogous reaction with neopentyl thiol 16 was precluded by the thiophilicity of silver. However, we found that the corresponding thiolate $(\mathrm{NaH})$ rapidly displaced the bromide in trisaccharide donor 20 to give glycosyl thioether $\mathbf{2 2}$ with complete $\beta$-selectivity.

\section{Construction of glycosidic linkages using the linear trisaccharide glycosyl donor as a nucleophile}

While the natural $\beta$-glycosyl ester linkage in the lead compound 2 (SQS-0-0-5-5) can be accessed by Schmidt glycosylation of the C28-carboxylic acid in PPS 10 with the corresponding trisaccharide trichloroacetimidate donor, ${ }^{21}$ analogous efforts to synthesize thioester and $\alpha$-ester linkages using a variety of traditional glycosylation methods (Lewis acid-catalyzed displacement of glycosyl donors, dehydrative glycosylations, displacement of glycosyl bromides under phase transfer conditions) led to complex mixtures of anomers and other unidentified impurities. Thus, to access these QS-21 analogues, as well as the corresponding carbamates, we used a conceptual reverse of polarity, with the glycoside acting as a nucleophile (Scheme 3). First, treatment of trisaccharide hemiacetal donor 17 with $\mathrm{NaH}$ followed by addition of prosapogenin isocyanate 11 afforded carbamate-linked intermediates $\boldsymbol{\beta}-\mathbf{2 3}$ and $\boldsymbol{\alpha}-\mathbf{2 3}$ as an easily separable mixture of anomers $(2: 1 \beta / \alpha, 79 \%$ combined yield). In contrast, acylation of hemiacetal donor 17 with prosapogenin acid chloride $\mathbf{1 2}$ could be achieved with preferential formation of the $\alpha$-glycosyl ester intermediate $24(6: 1 \alpha / \beta, 70 \%$ combined yield of separable anomers). Conversely, addition of $\mathrm{NaH}$ to a solution of prosapogenin acid chloride $\mathbf{1 2}$ and trisaccharide thiohemiacetal $\mathbf{2 5}$ (generated by displacement of bromide in $\mathbf{2 0}$ with thioacetate, followed by deacetylation) furnished $\beta$-glycosyl thioester intermediate 26 in $87 \%$ yield with complete anomeric selectivity. Although all three of these linkages were formed under similar reaction conditions $(\mathrm{NaH}$; acylation), the diverse anomeric selectivities can be attributed to the anomeric ratio of the corresponding hemi(thio)acetal substrates and solvent effects. ${ }^{26}$

\section{Installation of acyl chains and global deprotection of QS saponin linkage variants}

With all of the desired glycosylated intermediates $(\mathbf{1 8}, \mathbf{1 9}, \mathbf{2 1 - 2 4}$, 26) in hand, each was advanced to the corresponding QS saponin central linkage variant (3-9) in a three-step sequence involving: (1) azide reduction $\left(\mathrm{H}_{2} \mathrm{~S}, \mathrm{Et}_{3} \mathrm{~N}\right),(2)$ acylation of the resulting amine with dodecanedioic acid monobenzyl ester, and (3) global deprotection via hydrogenolysis $\left(\mathrm{H}_{2}, \mathrm{Pd} / \mathrm{C}\right)$ and acid hydrolysis $\left(\mathrm{TFA} / \mathrm{H}_{2} \mathrm{O}\right)$, followed by HPLC purification (Scheme 4). 


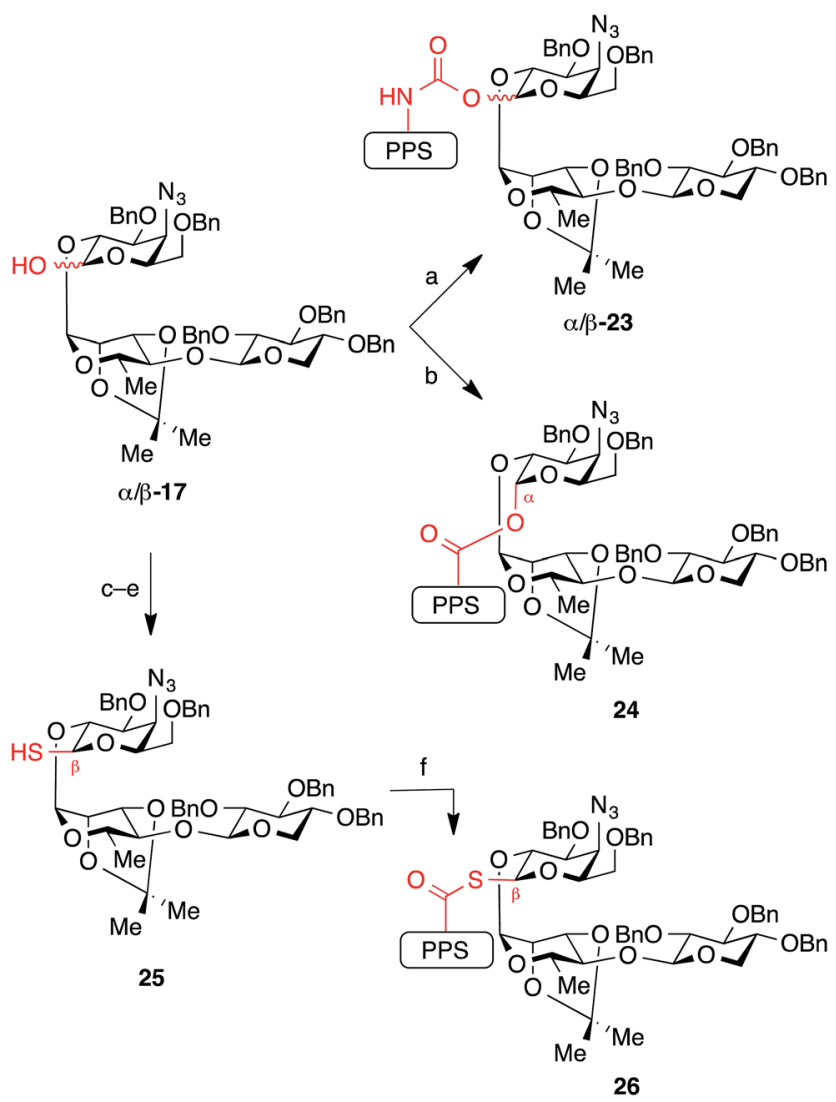

Scheme 3 Synthesis of glycosidic linkage variants using the glycosyl donor as a nucleophile. Reagents and conditions: (a) $\mathrm{NaH}$, then add 11 THF, 79\% combined yield of separable anomers (2 : $1 \beta / \alpha: 53 \% \boldsymbol{\beta}-23$, $26 \% \alpha-23)$; (b) $\mathrm{NaH}$, then add $12,1: 1 \mathrm{THF} / \mathrm{DMF},-20{ }^{\circ} \mathrm{C}, 70 \%(6: 1 \alpha / \beta$ : $60 \% \alpha-24,10 \% \beta-24)$; (c) $(\mathrm{COBr})_{2}, \mathrm{DMF}, \mathrm{TBP}, \mathrm{CH}_{2} \mathrm{Cl}_{2}, 0{ }^{\circ} \mathrm{C}, 80 \%$; (d) $\mathrm{Cs}_{2} \mathrm{CO}_{3}, \mathrm{AcSH}, 1: 1 \mathrm{THF} / \mathrm{DMF}, 87 \%$ ( $\beta$ only); (e) $\mathrm{H}_{2} \mathrm{NNH}_{2}, \mathrm{DTT}, 1: 1$ $\mathrm{MeOH} / \mathrm{THF}, 94 \%$; (f) $12, \mathrm{NaH}, \mathrm{THF}, 0{ }^{\circ} \mathrm{C}, 87 \%$ ( $\beta$ only). DTT $=$ dithiothreitol.

\section{Preclinical evaluation of QS saponin linkage variants in} a mouse vaccination model

We next sought to investigate the adjuvant activity of these linkage variants in comparison to SQS-21 (synthetic QS-21, 2 : 1 mixture of $\mathbf{1 a} / \mathbf{1} \mathbf{b})$ and the parent $\beta$-glycosyl ester lead compound 2 (SQS-0-0-5-5). There is currently no in vitro method to measure adjuvant activity, due in part to the unknown and likely multivariate mechanisms of saponin adjuvant action. Thus, we proceeded directly to testing these QS variants in vivo in a mouse vaccination model. Cohorts of mice were immunized with the saponin variant of interest ( 5 or $20 \mu \mathrm{g}$ ) and a four-antigen cocktail comprising: (1) the immunogenic peptide MUC1 (prostate and breast cancer antigen, non-glycosylated tandem repeat) conjugated to a highly immunogenic carrier protein KLH (keyhole limpet hemocyanin; MUC1-KLH), (2) the poorly immunogenic ganglioside GD3 (melanoma, neuroblastoma, sarcoma antigen) conjugated to KLH (GD3-KLH), and (3) the immunogenic protein antigen ovalbumin (OVA). ${ }^{20-22,27}$ The antibody titers elicited against all four antigens were evaluated by ELISA to compare the adjuvant activity of each QS analogue (Fig. 2, S1, and $\mathrm{S} 2 \dagger) .{ }^{28}$ As a general measure of adjuvant toxicity, percent weight loss over the first week after immunization was also monitored for each mouse (Fig. S3†).

Among the linker length variants, which feature longer distances between the triterpene and linear oligosaccharide domains, $\beta$-glycosyl ethanolamide 3 (SQS-0-4-5-5) and $\beta$-glycosyl carbamate $\boldsymbol{\beta}-\mathbf{4}$ (SQS-0-5-5-5), were inactive as adjuvants, inducing antibody titers against all four antigens that were not significantly higher than the no-adjuvant negative control. ${ }^{29}$ This marked decrease in adjuvant activity indicates that the central glycosidic linkage is less tolerant to modification than the other domains of the QS saponins..$^{20-22}$ In contrast, the $\beta$ glycosyl thioester analogue 5 (SQS-0-13-5-5) showed overall efficacy equal to or greater than that of both SQS-21 (1a/1b) and the lead compound 2 (SQS-0-0-5-5) (Fig. 2). Dose-limiting toxicity was observed at the higher $20 \mu \mathrm{g}$ dose (Fig. S3†), with

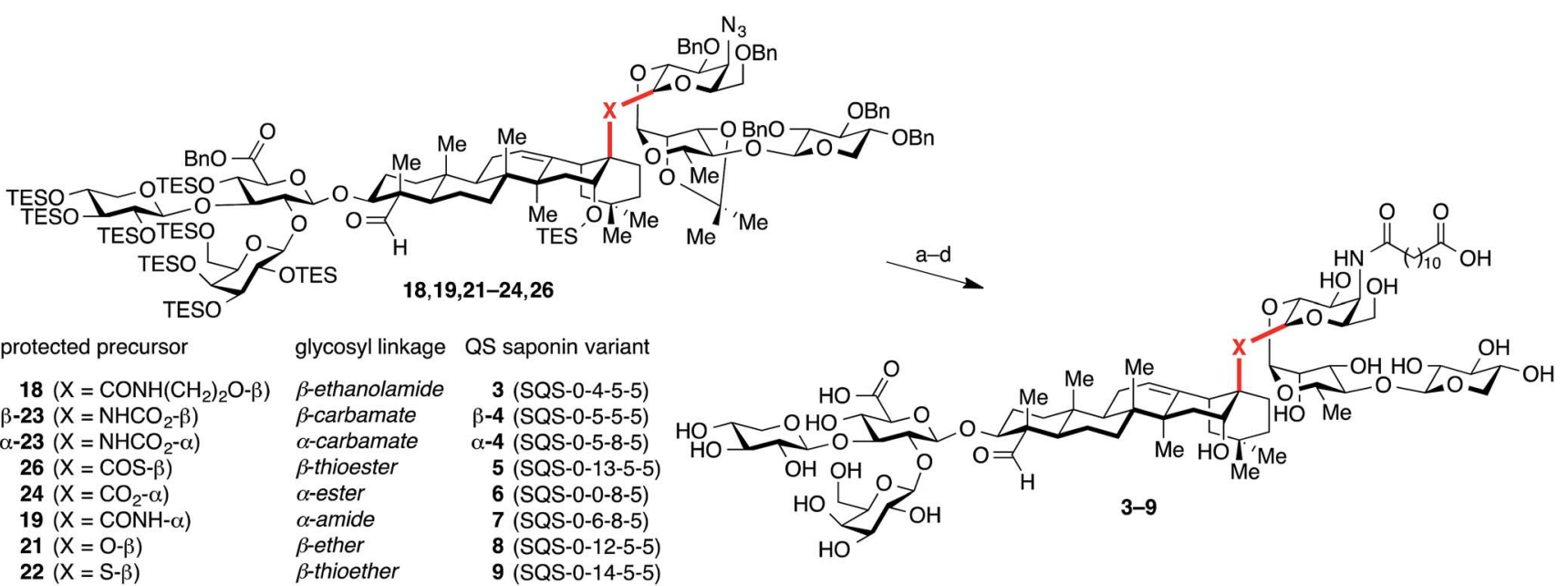

Scheme 4 Installation of acyl chains and global deprotection of QS saponin linkage variants. Reagents and conditions (a) $\mathrm{H}_{2} \mathrm{~S}, \mathrm{pyr}, \mathrm{Et} \mathrm{H}_{3} \mathrm{~N}, 78-94 \%$; (b) dodecanedioic acid monobenzyl ester, $i$-BuOCOCl, Et ${ }_{3} \mathrm{~N}, \mathrm{THF}, \mathrm{O}^{\circ} \mathrm{C}, 4 \mathrm{~h}$, then add appropriate amine from step a, 57-93\%; (c) $\mathrm{H}_{2}, \mathrm{Pd} / \mathrm{C}, 1: 1$ THF/EtOH, 4-16 h; (d) $3: 1 \mathrm{TFA} / \mathrm{H}_{2} \mathrm{O}, 0{ }^{\circ} \mathrm{C}, 1 \mathrm{~h}, 33-82 \%$ yield (two steps). 
(a)

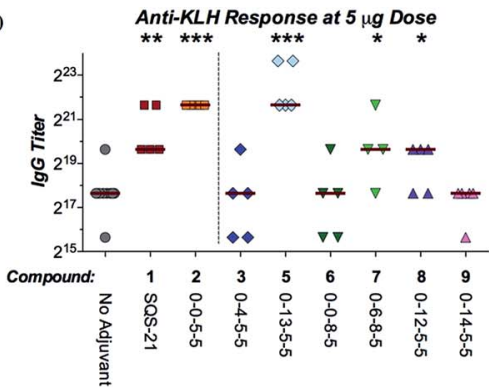

(d)

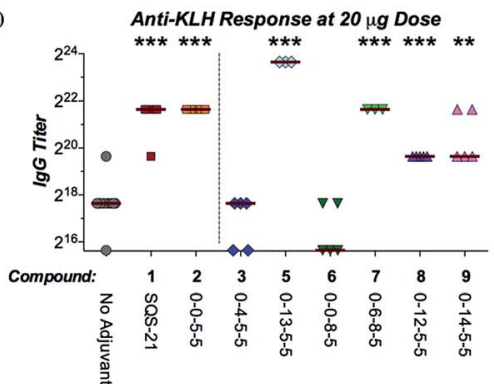

(b)

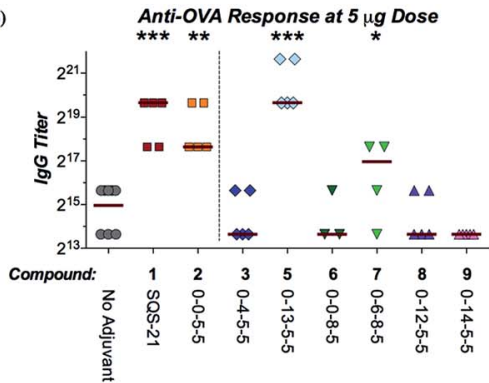

(e)

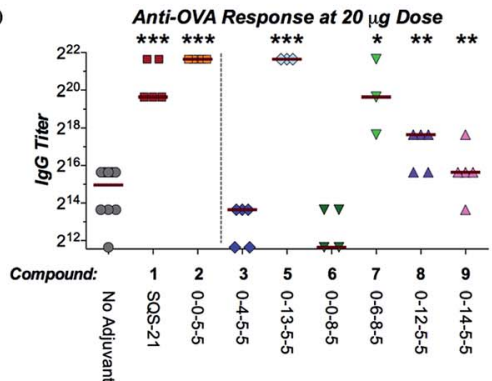

(c)

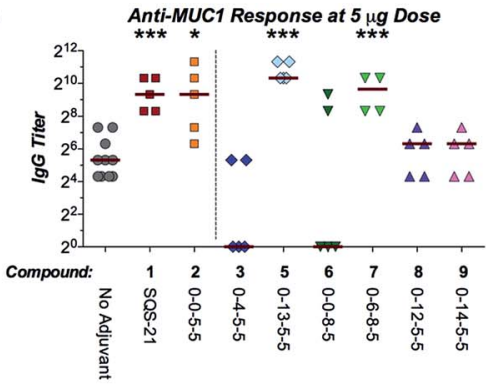

(f)

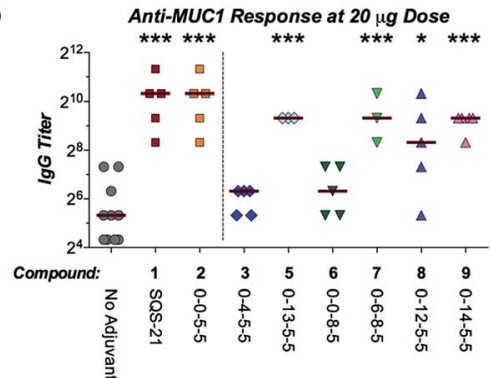

Fig. 2 Adjuvant activity of QS saponin linkage variants in a preclinical mouse vaccination model. (a) Anti-KLH (lgG), (b) anti-OVA (lgG), and (c) anti-MUC1 (IgG) antibody titers at $5 \mu \mathrm{g}$ dose of each saponin variant. (d) Anti-KLH (lgG), (e) anti-OVA (IgG), and (f) anti-MUC1 (IgG) antibody titers at $20 \mu \mathrm{g}$ dose of each saponin variant. See Fig. S1 and S2 $\uparrow$ for anti-GD3 antibody titers. $\alpha / \beta$-Glycosyl carbamates $\alpha / \beta-4$ were inactive at both doses (not shown). Median titer values represented as horizontal bars. Statistical significance compared to the no-adjuvant negative control assessed using unpaired Student's $t$-test with $\mathrm{Cl}=95 \%$ : ${ }^{*} p \leq 0.05, * * p<0.01, * * *<0.001$.

two out of five mice in this cohort dying seven days after vaccination (Table S1 $\dagger$ ). However, no weight loss was observed at the $5 \mu \mathrm{g}$ dose (Fig. S3†), at which the glycosyl thioester retained potent adjuvant activity. Among the stereoisomeric $\alpha$ linked variants, the $\alpha$-glycosyl ester 6 (SQS-0-0-8-5) ${ }^{30}$ and $\alpha$ glycosyl carbamate $\boldsymbol{\alpha - 4}$ (SQS-0-5-8-5) were also inactive as adjuvants with all four antigens. It is striking that a change in a single stereocenter ( $\alpha$-glycosyl ester 6 vs. $\beta$-glycosyl ester 2 ) has such a dramatic effect upon the adjuvant activity of these large, complex molecules. Interestingly, replacement of the $\alpha$-glycosyl ester in 6 with an $\alpha$-glycosyl amide in 7 (SQS-0-6-8-5) restored adjuvant activity against KLH and MUC1 at the $5 \mu \mathrm{g}$ dose, and against all four antigens at the $20 \mu \mathrm{g}$ dose. However, acute toxicity emerged at both doses (Fig. S3†), with one out of five mice in each cohort dying within a week of vaccination (Table $\mathrm{S} 1 \dagger$ ).

Along these lines, the $\beta$-glycosyl ether variant 8 (SQS-0-12-5-5) and $\beta$-glycosyl thioether variant 9 (SQS-0-14-5-5), in which the lack of the carbonyl group compared to $\beta$-glycosyl ester 2 and $\beta$ glycosyl thioester 5, respectively, affords increased conformational flexibility between the triterpene and linear oligosaccharide domains, exhibited intermediate adjuvant efficacy, with little or no activity observed at the $5 \mu \mathrm{g}$ dose but significant activity against KLH, OVA, and MUC1 at the $20 \mu \mathrm{g}$ dose. Both variants exhibited negligible toxicity at both doses (Fig. S3†). The increased conformational flexibility in the linkage between the triterpene and linear oligosaccharide domains may provide a molecular rationale for this attenuated activity (vide infra).

One of the limitations of the natural product QS-21 is the chemical instability of the esters in the acyl chain domain in aqueous solution, which is also associated with its toxicity. ${ }^{20}$
Although $\beta$-glycosyl thioester 5 does not contain these labile esters, we assessed potential chemical instability at other sites, such as the glycosyl thioester linkage, using a reported accelerated stability assay, ${ }^{31}$ designed to mimic suboptimal storage conditions in the developing world. While incubation of QS-21 at $37{ }^{\circ} \mathrm{C}$ in phosphate buffered saline $(\mathrm{pH}$ 7.0) results in degradation to $90 \%$ purity in 1.2 days $\left(t_{90}\right),{ }^{31} \beta$-glycosyl thioester 5 exhibited no measurable degradation (HPLC, ${ }^{1} \mathrm{H}-\mathrm{NMR}$, data not shown) over seven days under these conditions.

\section{Conformational analysis of saponin variants using molecular dynamics simulations}

To seek a molecular rationale for the striking variation in immunoadjuvant activity observed for these saponin variants, we performed $200 \mathrm{~ns}$ molecular dynamics simulations on the variants and parent natural product QS-21-Api (1a) in explicit water (TIP3P model) with explicit counterions $\left(\mathrm{Na}^{+}\right)$to neutralize each system, using the AMBER ff14SB, gaff, and GLYCAM force-fields. ${ }^{32}$ Because the formation of micelles is not essential for the observed adjuvant activity, ${ }^{33,34}$ solvated single molecules were analyzed in the simulations.

Strikingly, the central linkage variants exhibited clear conformational differences that correlated with relative adjuvant activity (Fig. 3). In general, the most potent saponins adopted a conformation in which the acyl chain (gray) was folded back over the triterpene core (green), between the branched trisaccharide (magenta) and linear oligosaccharide (orange) domains. The local conformation about the central linkage and extent of folding were characterized quantitatively in two ways, by measuring the torsional angles about the central linkage (i.e.: 

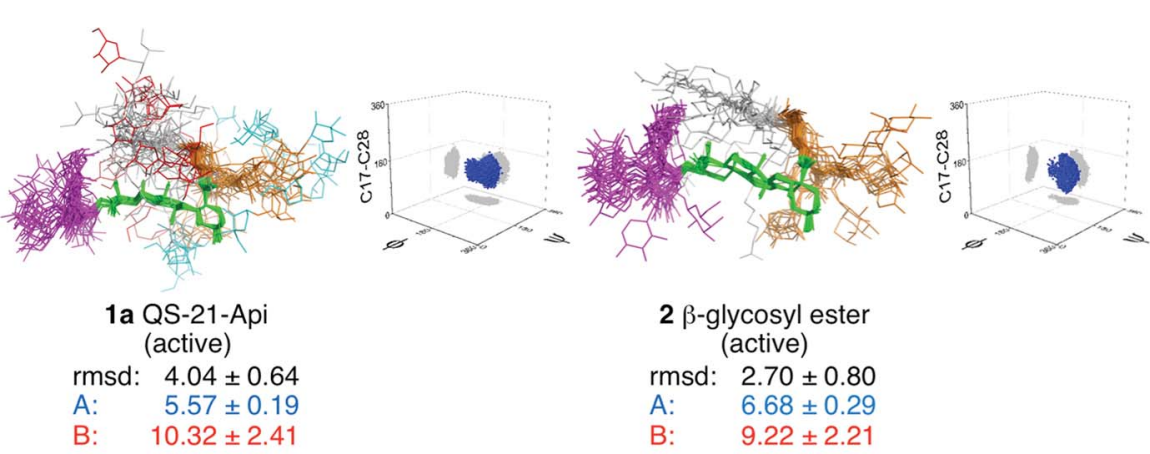
$2 \beta$-glycosyl ester (active)
rmsd: $\quad 2.70 \pm 0.80$
A: $\quad 6.68 \pm 0.29$
B: $\quad 9.22 \pm 2.21$
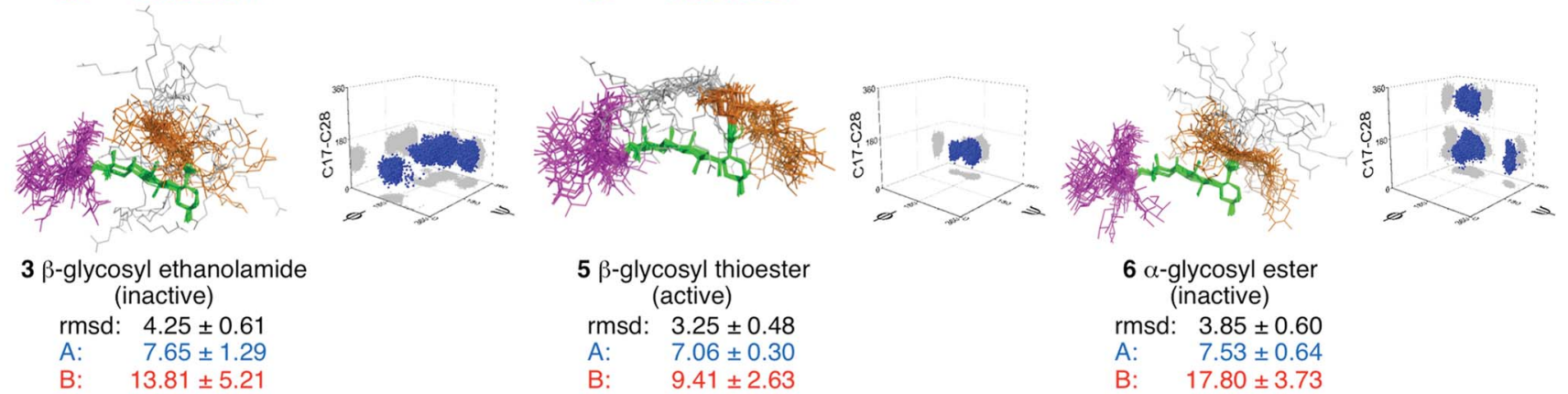

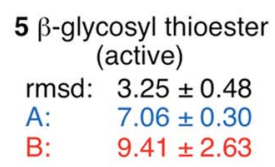
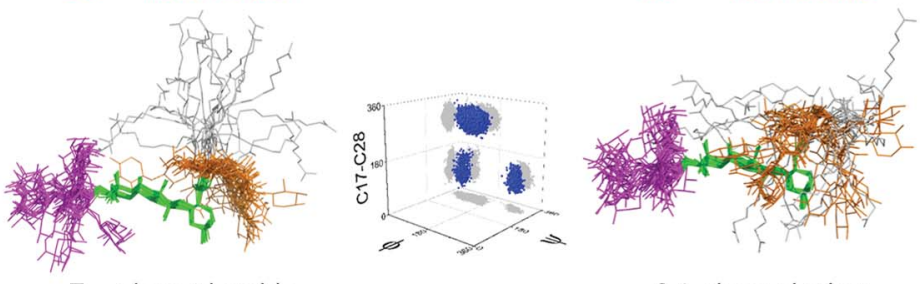

$7 \alpha$-glycosyl amide (attenuated)

rmsd: $2.82 \pm 0.68$

A: $\quad 7.62 \pm 0.59$

B: $\quad 16.90 \pm 3.93$

$8 \beta$-glycosyl ether (attenuated)

rmsd: $\quad 4.04 \pm 0.63$

A: $\quad 8.14 \pm 0.68$

B: $\quad 13.00 \pm 4.32$

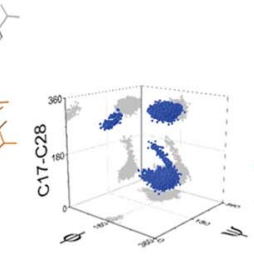

$6 \alpha$-glycosyl ester (inactive)

rmsd: $\quad 3.85 \pm 0.60$

A: $\quad 7.53 \pm 0.64$

B: $\quad 17.80 \pm 3.73$

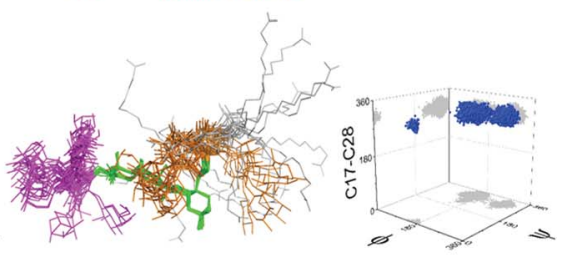

$9 \beta$-glycosyl thioether (attenuated)

rmsd: $\quad 4.27 \pm 0.67$

A: $\quad 7.88 \pm 0.59$

B: $\quad 15.80 \pm 4.81$
QS-21-Api

$A=$ distance between triterpene $\mathrm{B}$-ring centroid bridging monosaccharide centroid

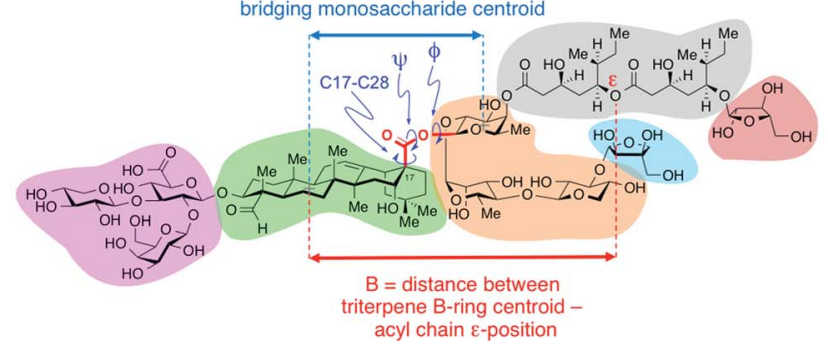

QS saponin central glycosidic linkage variants

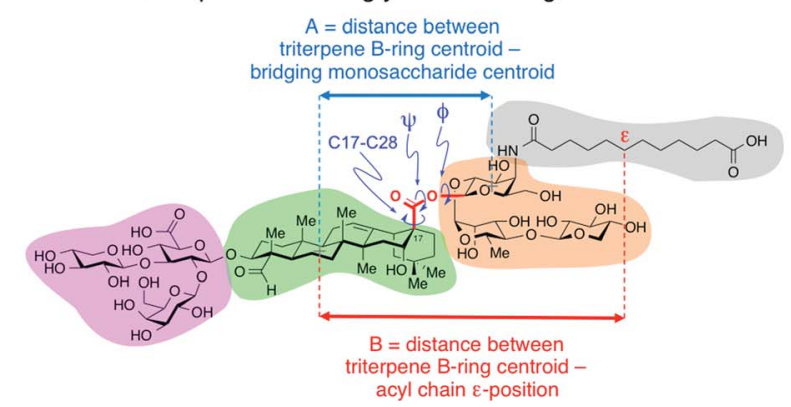

Fig. 3 Ensembles obtained from unrestrained 200 ns molecular dynamics simulations of QS-21-Api (1a) and saponin variants 2, 3, and 5-9. The rmsd $(\AA)$ for heavy atoms relative to average structure, key distance constraints $(\AA)$, and three-dimensional plots of torsional angle distributions $(\mathrm{C} 17-\mathrm{C} 28, \psi, \varphi$, arbitrary scale $\dagger)$ about the central glycosidic linker are shown. QS saponin structural domains are color-coded: branched trisaccharide (magenta), triterpene (green), linear trisaccharide (orange), acyl chain (gray), terminal sugars (QS-21 only: cyan, red). In the threedimensional plots, torsional angle distributions are shown in blue and projections onto each plane are shown in gray. Active adjuvants exhibit distinctive and tightly-clustered torsional angle distributions about the central glycosidic linkage and comparatively shorter distances between the centroid of the triterpene $B$-ring and the centroid of the bridging monosaccharide ( $A$, blue) as well as between the centroid of the triterpene B-ring and the acyl chain $\varepsilon$-position (B, red). See Fig. S4-S8† for additional details.

C17 of the triterpene core to $\mathrm{C} 1$ of the bridging monosaccharide of the linear oligosaccharide domain), and by determining the mean distances between the centroid of triterpene B-ring and two points on the saponin: the $\varepsilon$ position of the acyl chain and the centroid of the bridging monosaccharide (see also Fig. S4†).
The central linkage torsional angles for active saponins QS-21Api (1a), $\beta$-glycosyl ester 2, and $\beta$-glycosyl thioester 5 were strikingly similar and clustered tightly, effectively defining the position and orientation of the bridging monosaccharide with its $\beta$-face approximately perpendicular to the rear edge of the 
triterpene (Fig. 3 and $55 \dagger$ ). This orientation places the acyl chain where it can be folded over the triterpene. This folded conformation was most clearly observed in the two most active synthetic saponins, $\beta$-glycosyl ester 2 and $\beta$-glycosyl thioester 5. Notably, the most highly populated conformations of the parent natural product QS-21-Api (1a) also featured a similar orientation of the bridging monosaccharide and presentation of the acyl chain relative to the triterpene and flanking oligosaccharides. In contrast, the central linkage torsional angles and acyl chain exhibited less organized and considerably different relative orientations in the other saponin variants, with the acyl chain being folded over the triterpene to a much lesser degree in attenuated adjuvants ( $\alpha$-glycosyl amide 7, $\beta$-glycosyl ether 8, $\beta$-glycosyl thioether 9) and directed away from the triterpene in the inactive saponins ( $\beta$-glycosyl ethanolamide 3 and $\alpha$-glycosyl ester 6 ). Furthermore, in the most active adjuvants, the linear oligosaccharide domain (orange) was oriented away from the center of the triterpene in an extended orientation, as illustrated by $\beta$-glycosyl thioester 5, as well as $\beta$-glycosyl ester 2 and QS-21Api (1a). In contrast, attenuated and inactive adjuvants such as $\beta$-glycosyl ether 8, $\beta$-glycosyl thioether 9, $\beta$-glycosyl ethanolamide 3 , and $\alpha$-glycosyl ester 6 showed a distinct orientation for the linear oligosaccharide domain, which was more disorganized and folded towards the triterpene. Torsional angle distributions for all other glycosidic linkages were generally consistent with those observed in QS-21, ${ }^{32 a}$ independent of adjuvant activity (Fig. S6-S8†).

The molecular dynamic simulations above provided saponin conformations that were predictive of adjuvant activity. However, to assess potential conformational effects of other less abundant cations or localization at the cell membrane, which has been postulated to be the site of action for these saponins, we also performed molecular dynamic simulations of QS-21-Api (1a), active $\beta$-glycosyl ester 2 , and inactive $\alpha$-glycosyl ester 6 in which the $\mathrm{Na}^{+}$counterion was replaced with $\mathrm{K}^{+}$or $\mathrm{Mg}^{2+}$ (Fig. S9 $\dagger$ ), or in more hydrophobic trifluoroethanol solvent (Fig. S10 $\dagger^{\dagger}$ ). The active saponins $1 \mathrm{a}$ and 2 exhibited similar overall conformations under all of these conditions, distinct from those of the inactive saponin $\mathbf{6}$.

\section{Conclusions}

It has generally been assumed that immunopotentiation by a wide range of Quillaja and other saponin adjuvants ${ }^{35}$ is largely due to their general physicochemical properties as soap-like molecules that non-specifically induce a local inflammatory response, stimulating antigen-presenting cells and increasing antigen uptake, similar to the emulsion-based adjuvants. ${ }^{\mathbf{4} 6,36}$ However, the combined immunological and molecular dynamics results obtained with the synthetic QS-21 variants herein demonstrate that seemingly subtle changes in saponin structure can have dramatic effects upon the solution conformations of these molecules that correlate with their adjuvant activity. For example, relative to the lead compound 2, increasing linker length by one bond in $\beta$-glycosyl carbamate $\boldsymbol{\beta}-\mathbf{4}$ or three bonds in $\beta$-glycosyl ethanolamide 3 abrogates activity, as does changing a single stereogenic center in $\alpha$-glycosyl ester 6 . In fact, the latter variants exhibit considerably different conformations compared to 2, with less-organized and differently-oriented acyl chain and linear oligosaccharide domains relative to the triterpene core. In contrast, a more modest increase in the molecular distance between the triterpene and linear oligosaccharide domains provided the potent $\beta$-glycosyl thioester variant $\mathbf{5}$, which adopts a rigid, folded conformation, similar to glycosyl ester 2, in which the acyl chain is oriented approximately in parallel to the triterpene core.

Moreover, in the molecular dynamics simulations, these active variants adopted the narrowest range of conformations, exhibiting relatively rigid structures that are similar to the most heavily populated conformation calculated for the natural product QS-21-Api (1a). This raises the possibility of a preferred 'active conformation' for these potent adjuvants. Furthermore, increased conformational flexibility about the central glycosidic linkage in $\beta$-glycosyl ether $\mathbf{8}$ and $\beta$-glycosyl thioether $\mathbf{9}$ provides attenuated variants that adopt a wider range of conformations that are distinct from the proposed folded 'active conformation'. Thus, these molecular dynamics simulations have provided important insights into the conformational features of QS saponins that correlate with adjuvant activity, which may aid in the design of new analogues in the future.

We have previously shown that adjuvant-active fluorescent and radiolabeled QS saponin variants, but not structurallysimilar inactive congeners, are internalized into antigen presenting cells in vitro ${ }^{\mathbf{2 1 , 2 2}}$ and accumulate in the draining lymph nodes in vivo. ${ }^{22}$ These results, together with the correlation between three-dimensional structure and adjuvant activity observed herein, are consistent with the hypothesis that QS saponins may interact with a discrete molecular target as the basis for their adjuvant activity, in contrast to emulsion-based immunoadjuvants that are known to act through more general immunostimulatory processes. ${ }^{6}$

In conclusion, we have designed and synthesized a series of QS saponin variants to explore the effect of structural variations of the central glycosidic linkage on adjuvant activity, and have studied the implications of such modifications on saponin conformation by molecular dynamics simulations. Although the chemical complexity of these systems presented several synthetic challenges, we were able to access a number of analogues to probe the effects of linker distance, stereochemistry, and conformational flexibility upon in vivo adjuvant activity and toxicity. In the context of the overall saponin structure, these relatively conservative modifications led to striking differences in adjuvant activity, highlighting the junction of the triterpene and linear oligosaccharide domain as playing a critical role in the biological activity of the QS saponins. Notably, these minor structural perturbations also led to distinct conformational preferences in molecular dynamics simulations, providing a possible molecular rationale for the divergent adjuvant activities observed. In addition, $\beta$-glycosyl thioester analogue 5 (SQS-0-13-5-5) showed particularly potent adjuvant activity compared to SQS-21, the parent lead compound ( $\beta$-glycosyl ester 2, SQS-0-0-5-5), and all other linkage 
variants tested. In the future, it will be of interest to investigate further modifications of the central linker carbonyl (e.g., oxetane, cyclopropyl) and to develop tailored QS-21 variants that combine this thioester linkage modification with deletion of the left-hand branched trisaccharide domain, removal of which has recently been shown to reduce toxicity in vivo without impairing adjuvant activity. ${ }^{22}$ Moreover, molecular dynamics evaluation of the conformational features of other previously synthesized QS variants would provide further structural correlations that may enable the use of such computational simulations as a predictive platform for analogue design. Finally, the pronounced SAR established in this work is suggestive of interaction with a defined molecular receptor, setting the stage for additional studies to identify possible molecular targets of these promising immunoadjuvants.

\section{Conflict of interest}

The authors declare the following competing financial interest(s): G. R., and D. Y. G. are founders of and have financial interests in Adjuvance Technologies, Inc.

\section{Acknowledgements}

Dedicated to the memory of our mentor and colleague, Professor David Y. Gin (1967-2011). We thank Dr E. Chea, and Dr R. Karimov (MSKCC) for helpful discussions and Dr G. Sukenick, Rong Wang, Dr H. Liu, H. Fang, and Dr S. Rusli (MSKCC) for expert NMR and mass spectral support. Financial support from the European Commission (Marie Curie International Outgoing Fellowship to A. F.-T.) NIH (R01 AI085622 to G. R. and D. Y. G., R01 GM058833 to D. S. T., G. R., and D. Y. G., Cancer Center Support Grant P30 CA008748 to C. B. Thompson in support of the Center of Comparative Medicine \& Pathology and Clinical Grade Production Core Facility), William and Alice Goodwin and the Commonwealth Foundation for Cancer Research, and MSKCC Experimental Therapeutics Center is gratefully acknowledged.

\section{Notes and references}

1 G. Leroux-Roels, Vaccine, 2010, 28 (suppl. 3), C25-C36.

2 R. L. Coffman, A. Sher and R. A. Seder, Immunity, 2010, 33, 492-503.

3 W. C. Koff, D. R. Burton, P. R. Johnson, B. D. Walker, C. R. King, G. J. Nabel, R. Ahmed, M. K. Bhan and S. A. Plotkin, Science, 2013, 340, 1232910.

4 (a) S. L. Hem and H. HogenEsch, Expert Rev. Vaccines, 2007, 6, 685-698; (b) P. Marrack, A. S. Mckee and M. W. Munks, Nat. Rev. Immunol., 2009, 9, 287-293.

5 N. Garcon, P. Chomez and M. van Mechelen, Expert Rev. Vaccines, 2007, 6, 723-739.

6 (a) D. T. O'Hagan, G. S. Ott, E. de Gregorio and A. Seubert, Vaccine, 2012, 30, 4341-4348; (b) D. T. O'Hagan, G. S. Ott, G. van Nest, R. Rappuoli and G. Del Giudice, Expert Rev. Vaccines, 2013, 12, 13-30.
7 G. Del Giudice and R. Rappuoli, Curr. Top. Microbiol. Immunol., 2015, 386, 151-180.

8 A. M. Didierlaurent, S. Morel, L. Lockman, S. L. Giannini, M. Bisteau, H. Carlsen, A. Kielland, O. Vosters, N. Vanderheyde, F. Schiavetti, D. Larocque, M. van Mechelen and N. Garcon, J. Immunol., 2009, 183, 6186-6197.

9 G. van Kriekinge, X. Castellsague, D. Cibula and N. Demarteau, Vaccine, 2014, 32, 733-739.

10 G. Gefenaite, M. Tacken, J. Bos, I. Stirbu-Wagner, J. C. Korevaar, R. P. Stolk, B. Wolters, M. Bijl, M. J. Postma, J. Wilschut, K. L. Nichol and E. Hak, PLoS One, 2013, 8, e66125.

11 (a) C. R. Kensil, U. Patel, M. Lennick and D. Marciani, J. Immunol., 1991, 146, 431-437; (b) M. J. Newman, J. Y. Wu, B. H. Gardner, K. J. Munroe, D. Leombruno, J. Recchia, C. R. Kensil and R. T. Coughlin, J. Immunol., 1992, 148, 2357-2362.

12 G. Ragupathi, J. R. Gardner, P. O. Livingston and D. Y. Gin, Expert Rev. Vaccines, 2011, 10, 463-470.

13 The RTS, S Clinical Trials Partnership, PLoS Med., 2014, 11, e1001685.

14 J. L. Cleland, C. R. Kensil, A. Lim, N. E. Jacobsen, L. Basa, M. Spellman, D. A. Wheeler, J. Y. Wu and M. F. Powell, J. Pharm. Sci., 1996, 85, 22-28.

15 C. R. Kensil and R. Kammer, Expert Opin. Invest. Drugs, 1998, 7, 1475-1482.

16 G. C. Kite, M. J. Howes and M. S. Simmonds, Rapid Commun. Mass Spectrom., 2004, 18, 2859-2870.

17 (a) D. J. Marciani, Drug Discovery Today, 2003, 8, 934-943; (b) S. Soltysik, J. Y. Wu, J. Recchia, D. A. Wheeler, M. J. Newman, R. T. Coughlin and C. R. Kensil, Vaccine, 1995, 13, 14031410.

18 (a) Y. J. Kim, P. F. Wang, M. Navarro-Villalobos, B. D. Rohde, J. Derryberry and D. Y. Gin, J. Am. Chem. Soc., 2006, 128, 11906-11915; (b) P. F. Wang, Y. J. Kim, M. NavarroVillalobos, B. D. Rohde and D. Y. Gin, J. Am. Chem. Soc., 2005, 127, 3256-3257.

19 K. Deng, M. M. Adams and D. Y. Gin, J. Am. Chem. Soc., 2008, 130, 5860-5861.

20 M. M. Adams, P. Damani, N. R. Perl, A. Won, F. Hong, P. O. Livingston, G. Ragupathi and D. Y. Gin, J. Am. Chem. Soc., 2010, 132, 1939-1945.

21 E. K. Chea, A. Fernandez-Tejada, P. Damani, M. M. Adams, J. R. Gardner, P. O. Livingston, G. Ragupathi and D. Y. Gin, J. Am. Chem. Soc., 2012, 134, 13448-13457.

22 A. Fernandez-Tejada, E. K. Chea, C. George, N. Pillarsetty, J. R. Gardner, P. O. Livingston, G. Ragupathi, J. S. Lewis, D. S. Tan and D. Y. Gin, Nat. Chem., 2014, 6, 635-643.

23 (a) B. A. Garcia, J. L. Poole and D. Y. Gin, J. Am. Chem. Soc., 1997, 119, 7597-7598; (b) Z. Györgydeák, Z. Hadady, N. Felföldi, A. Krakomperger, V. Nagy, M. Tóth, A. Brunyánszki, T. Docsa, P. Gergely and L. Somsák, Bioorg. Med. Chem., 2004, 12, 4861-4870.

24 (a) D. P. Galonic, W. A. van der Donk and D. Y. Gin, J. Am. Chem. Soc., 2004, 126, 12712-12713; (b) M. J. Bourgeois, D. Gueyrard, E. Montaudon and P. Rollin, Lett. Org. Chem., 2005, 2, 665-667. 
25 The corresponding $\beta$-glycosyl pivalamide 27 was synthesized analogously from trisaccharide donor $17\left(\mathrm{Ph}_{2} \mathrm{SO}, \mathrm{Tf}_{2} \mathrm{O}, \mathrm{TBP}\right.$, DCM, $-45{ }^{\circ} \mathrm{C}$, then pivalamide, $78 \%$ ).

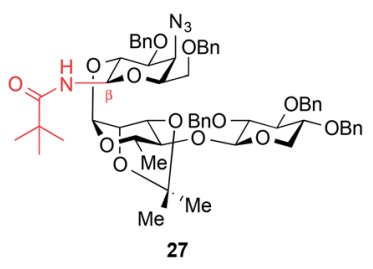

26 Schmidt and co-workers [R. R. Schmidt, U. Moering and M. Reichrath, Tetrahedron Lett., 1980, 21, 3565-3568] have proposed that the sodium alkoxides of gluco- and galactopyranosides may adopt the alternative ${ }^{1} C_{4}$ conformation, in which 1,3,5-triaxial chelation of the sodium ion favors equilibration to the $\beta$-anomer. Hemiacetal $\boldsymbol{\alpha} / \boldsymbol{\beta}-\mathbf{1 7}$ was isolated in a variable anomeric ratio, somewhat favoring the $\alpha$ anomer $(1: 1$ to $2.5: 1 \alpha / \beta)$. In the synthesis of glycosyl carbamates 23, $\mathrm{NaH}$ deprotonation of hemiacetal $\mathbf{1 7}$ was carried out in THF, in which the sodium counteranion likely remains closely associated with the alkoxide, but the prolonged incubation time $(80 \mathrm{~min})$ prior to isocyanate addition favored the reversed $\beta$-glycosyl carbamate $(2: 1 \beta / \alpha)$, presumably due to anomeric equilibration of the alkoxide (conversely, shorter incubation times of approximately $10 \mathrm{~min}$ favored the $\boldsymbol{\alpha - 2 3}$ anomer in a $2: 1$ ratio). In the synthesis of glycosyl ester 24, NaH deprotonation of hemiacetal 17 was carried out in $1: 1 \mathrm{THF} / \mathrm{DMF}$, a more polar solvent system that better solvates the hemiacetal alkoxide, resulting in rapid equilibration ( $<5 \mathrm{~min}$ ) to the thermodynamically favored $\alpha$-anomer (due to the anomeric effect) [A. G. M. Barrett and B. C. B. Bezuidenhoudt, Heterocycles, 1989, 28, 209-212], thus leading to the highly enriched $\alpha$ glycosyl ester $24(6: 1 \alpha / \beta)$. Finally, in the preparation of glycosyl thioester 26, the hemithioacetal substrate 25 was synthesized as a single $\beta$-anomer, which, upon $\mathrm{NaH}$ deprotonation, and in contrast to the corresponding hemiacetal alkoxide, did not anomerize rapidly [R. Caraballo, L. Q. Deng, L. Amorim, T. Brinck and O. Ramstrom, J. Org. Chem., 2010, 75, 6115-6121], resulting in the $\beta$-glycosyl thioester 26 with complete $\beta$-selectivity.
27 G. Ragupathi, P. Damani, K. Deng, M. M. Adams, J. Hang, C. George, P. O. Livingston and D. Y. Gin, Vaccine, 2010, 28, 4260-4267.

28 See ESI for complete details. $\dagger$

29 Preliminary immunizations for both carbamates, $\boldsymbol{\alpha} / \boldsymbol{\beta}-\mathbf{4}$, at doses up to $50 \mu \mathrm{g}$ showed no response (data not shown).

30 The $\alpha$-ester 6 was also inactive at a $50 \mu \mathrm{g}$ dose in the preclinical mouse vaccination model (data not shown).

31 G. A. Beltz and C. Kensil, Compositions comprising the adjuvant QS-21 and polysorbate or cyclodextrin as excipient. PCT Int. Appl. WO1999010008 A1, Mar 4, 1999.

32 (a) C. Pedebos, L. Pol-Fachin, R. Pons, C. V. Teixeira and H. Verli, Molecules, 2014, 19, 3744-3760; (b) V. Hornak, R. Abel, A. Okur, B. Strockbine, A. Roitberg and C. Simmerling, Proteins: Struct., Funct., Bioinf., 2006, 65, 712-725; $(c) \quad$ K. N. Kirschner, A. B. Yongye, S. M. Tschampel, J. Gonzalez-Outeirino, C. R. Daniels, B. L. Foley and R. J. Woods, J. Comput. Chem., 2008, 29, 622-655.

33 (a) P. O. Livingston, S. Adluri, F. Helling, T. J. Yao, C. R. Kensil, M. J. Newman and D. Marciani, Vaccine, 1994, 12, 1275-1280; (b) J. B. Press, R. C. Reynolds, R. D. May and D. J. Marciani, in Studies in Natural Products Chemistry, ed. R. Atta ur, Elsevier, 2000, pp. 131-174, vol. 24, Part E; (c) D. J. Marciani, C. R. Kensil, G. A. Beltz, C. H. Hung, J. Cronier and A. Aubert, Vaccine, 1991, 9, 89-96. 34 The critical micelle concentration for QS-21 ranges from 51 to $500 \mathrm{mg} \mathrm{L} \mathrm{L}^{-1}$, depending on the specific buffer conditions: J. L. Cleland, C. R. Kensil, A. Lim, N. E. Jacobsen, L. Basa, M. Spellman, D. A. Wheeler, J. Y. Wu and M. F. Powell, J. Pharm. Sci., 1996, 85, 22-28.

35 K. Oda, H. Matsuda, T. Murakami, S. Katayama, T. Ohgitani and M. Yoshikawa, Biol. Chem., 2000, 381, 67-74.

36 Previous studies have suggested that the natural QS-21 acyl chain may facilitate more specific immunostimulatory processes via interactions with cellular membranes (ref. $17 b)$, but the adjuvant activity of variants with drastically simplified acyl chains highlights the non-specificity of such a process. It has also been proposed that the C4aldehyde substituent of QS-21 may interact with an unidentified molecular target by Schiff base formation (J. Rhodes, et al., Therapeutic potentiation of the immunesystem by costimulatory Schiff-base forming drugs, Nature, 1995, 377, 71), although we have recently discovered that this functionality is dispensable for adjuvant activity in several synthetic QS saponin analogues (ref. 22). 